Venous thrombosis involved 20 patients (90\%). They were deep in 16 patients, superficial in one patient and superficial and deep in 3 patients. It was recurrent in 14 patients.

The most common site of deep vein thrombosis was the lower limbs $(n=17)$. The others had unusual sites: inferior vena cava thrombosis $(n=$ $3)$, superior vena cava thrombosis $(n=2)$, sus-hepatic vein $(n=1)$ and upper limb thrombosis $(n=1)$. Pulmonary embolism was noted in one case.

Arterial damage involved 4 patients (20\%) divided into arterial aneurysms ( 3 cases) and arterial thromboses ( 2 cases). The locations were divided as follows: lower extremity arteries in 2 cases, upper limb arteries in one case, pulmonary artery in 2 cases and abdominal aorta in one case. Lower limb ischemia had occurred in one patient.

Two patients (10\%) had mixed vascular, arterial and venous involvement, confirming the multiple and ubiquitous nature of angio-Behçet.

The treatment was based on colchicine, anticoagulants and corticosteroids in venous thromboses. Immunosuppressive therapy was started in 4 patients in front of the unusual site.

In arterial cases, corticosteroids in combination with immunosuppressant were prescribed. Flattening of the aneurysm was indicated in 2 cases with simple operative follow-up.

Conclusion: Behçet disease (BD) is very common in the Mediterranean basin, mainly affects the 30 -year-old man. This systemic disease is characterized by oral aphthosis, genital ulcers and systemic involvement including occular, gastrointestinal, neurological, and vessels that make the severity of the disease.

All types of vessels, regardless of size and seat, may be affected, with venous tropism.

Our study has just supported current literature data regarding the extensive and recurrent venous thrombosis during MB. The seriousness of this venous involvement lies in the involvement of the cavernous veins and in pulmonary embolism.

Arterial damage, such as thrombosis and/or aneurysm, is rare but maybe life-threatening.

Early diagnosis, intensive and appropriate treatment, regular follow-up and the involvement of a multidisciplinary team including internists, vascular surgeons and radiologists are key to better management of patients with angio -Behçet.

Our study illustrates the frequency and significance of vascular involvement in BD.

Disclosure of Interests: None declared

DOI: 10.1136/annrheumdis-2019-eular.5414

\section{AB1269 RISK FACTORS ASSOCIATED WITH PERIPHERAL NEUROPATHY (PN) IN ANCA-ASSOCIATED VASCULITIS (AAV):}

Violeta Higuera Ortiz ${ }^{1}$, Luis Humberto Silveira Torre ${ }^{2}$, Antonio Villa Romero ${ }^{3}$, Luis Felipe Flores-Suárez ${ }^{4} .{ }^{1}$ IMSS, Mexico City, Mexico; ${ }^{2}$ Instituto Nacional de Cardiología Ignacio Chávez, Mexico City, Mexico; ${ }^{3}$ Faculty of Medicine, UNAM, Mexico City, Mexico; ${ }^{4}$ Instituto Nacional de Enfermedades Respiratorias, Primary Systemic Vasculitides Clinic, Mexico City, Mexico

Background: Few studies have reported on the risk factors associated with PN in AAV. Vasculitic PN in AAV has been associated with male sex, arthritis, cutaneous, mucous membranes, and ENT manifestations of vasculitis $^{1,2,3}$. PN can lead to life quality impairment, as only $35 \%$ of the patients with PN have complete symptoms resolution within 6 months $^{1}$. Objectives: Along with describing the prevalence of incidence of PN in our cohort, we undertook this study to identify the risk factors associated with PN in AAV.

Methods: From 153 AAV patients (defined according to the EMA algorithm), 97 were eligible (complete data and no exclusion criteria -diabetes mellitus, axial disc disease, malignancies, herpetic neuralgia, other comorbidities). Clinical, electromyographic (EMG) and histopathological data were recorded. According to the presence (cases) or absence (controls) of clinical data for PN descriptive analysis is reported as means \pm SD or medians (interquartile range-IQR). Categorical variables are reported as percentages. Student t-test and Mann-Whitney U-test were used for continuous variables, two-tailed Fisher exact test to compare proportions differences. To estimate PN predictors, bivariate and multivariate models to establish hazard ratios [HR] were built and analysed with Cox regression Results: Thirty-two (33\%) patients (19-GPA, 9-MPA, 4-EGPA; 17 prevalent, 15 incident) had PN. Mean age \pm SD was $47.2 \pm 13.0$ at vasculitis diagnosis. The median follow-up was 55 months (IQR 37-79) until PN diagnosis. Main symptoms: paresthesias $97 \%$, hypoesthesia $72 \%$, hyporeflexia $53 \%$, burning pain and weakness $47 \%$, mononeuritis multiplex $44 \%$.
Diagnostic EMG support in $60 \%$. The estimated incidence rate of PN was of 4/100 persons-year. Differences between cases (n-32) and controls ( $n-65)$ were smoking history $(p=0.03)$, drug abuse $(p=0.04)$, and a higher mean BVAS $(\mathrm{p}<0.001)$ at AAV diagnosis. The table shows the factors associated with a higher risk for the development of PN. After adjustment for covariates and in a multivariate analysis, a higher BVAS at baseline conferred risk for the presence of $\mathrm{PN}(\mathrm{HR}=1.1,95 \% \mathrm{Cl} 1.03$ $1.25, \mathrm{p}=0.01)$.

Table. Risk factors for development of PN in AAV expressed as hazard ratios after Cox regression analysis.

\begin{tabular}{lcc}
\hline Variable & HR $(95 \% \mathrm{Cl})$ & $p$ value \\
\hline Drug abuse history & $4.34(1.2-15.6)$ & 0.025 \\
Arthritis & $2.98(1.01-8.7)$ & 0.04 \\
BVAS at AAV diagnosis & $1.13(1.03-1.23)$ & 0.005 \\
\hline
\end{tabular}

Conclusion: The risk for development of $\mathrm{PN}$ is related to overall higher disease activity when AAV is diagnosed. However, it may not be present after many years of its appearance. We had almost the same amount of incident cases than prevalent ones at diagnosis. Therefore, patients with a highly active disease need to be followed up specifically in order to detect $\mathrm{PN}$ during the disease course, probably with specialised neurological evaluation.

\section{REFERENCES}

[1] - Suppiah R, Hadden RDM, Batra R, et al. Peripheral neuropathy in ANCA-associated vasculitis: outcomes from the European Vasculitis Study Group trials. Rheumatology. 2011;50:2214-22.

[2] - de Groot K, Schimdt DK, Arlt AC, et al. Standarized Neurologic Evaluations of 128 Patients With Wegener Granulomatosis. Arch Neurol. 2001;58:1215-21.

[3] - Nishino H, Rubino FA, DeRemee RA, et al. Neurological involvement in Wegener's granulomatosis: an analysis of 324 consecutive patients at the Mayo Clinic. Ann Neurol 1993;33:4-9.

Disclosure of Interests: None declared

DOI: 10.1136/annrheumdis-2019-eular.4440

\section{AB1270 EVALUATIONS OF ANTIRHEUMATIC DRUGS AT PRECONCEPTIONAL, PREGNANCY AND POSTPARTUM PERIODS OF RA PATIENTS' IN A UNIVERSITY HOSPITAL; PRELIMINARY RESULTS}

Nesrin Caglayan Duman ${ }^{1}$, Atila Karaalp ${ }^{1}$, Nevsun Inanc ${ }^{2}$. 'Marmara University, School of Medicine, Department of Pharmacology, Istanbul, Turkey; ${ }^{2}$ Marmara University, School of Medicine, Division of Rheumatology, Department of Internal Medicine, Istanbul, Turkey

Background: Rheumatoid arthritis (RA) spontaneously improves during pregnancy and disease activity decreases in approximately two-thirds of pregnant but this decrease does not last long and postpartum exacerbation can be seen ${ }^{1}$.Conversely discontinuation of antirheumatic drug (ARD) during pregnancy may be a risk factor for exacerbation of $R^{2}$. In case of preconceptional, pregnancy and postpartum (P\&P\&P) situations during RA treatment, current options are discontinuation of inappropriate medications and switching to appropriate ones. With the latest BSR and EULAR guidelines on prescribing drugs in $\mathrm{P} \& \mathrm{P} \& \mathrm{P}$, treatment options expanded with some of the biologic drugs ${ }^{3,4}$.

Objectives: The aim of this study is to analyse alterations of ARD use (discontinuation, increasing or switching) at P\&P\&P periods of RA patients' retrospectively between 2002-2018.

Methods: In our records there are 25 cases of 19 female RA patients who having at least one pregnancy experienced out of 140 RA patients. The data were collected by telephone calls and patient file. Female patients that had no pregnancies after the RA diagnosis were not included. Preconception period was defined as 1 year before estimated last menstrual date. Postpartum period was defined as 1 year after infants' birth.

Results: RA patients participating in our study of 140 female (age median 55 (26-87)), 121 of them (86\%) did not give birth after the RA diagnosis. 19 of them (age median 39 (26-61)) had been pregnant after the RA diagnosis $(\% 14)$ and 14 of them gave birth 1,4 of them gave birth to 2 , one of them gave birth to 3 . Twelve out of $140(9 \%)$ patients had postnatal diagnosis. Each of the pregnancies counted as one case so some female patients represented in our data more than ones. While 22 of cases used the ARD before pregnancy (88\%), it decreased 18 of 